\title{
Study on the Risk Analysis and Emergency Management System of Major Accidents in Chemical Industry Park
}

Changxing Ren ${ }^{1}$, Haitao $\mathrm{Li}^{2}$, Ning Zhou ${ }^{2}$, Xiongjun Yuan ${ }^{2}$

1. Tianjin Fire Research Institute of the Ministry of Public Security, Tianjin 300381,China 2.Jiangsu Provincial Key Laboratory of oil and gas storage and transportation,College of Petroleum Engineering, Changzhou University, Jiangsu Changzhou 213016, China

\section{化工园区重大事故风险分析与应急管理体系研 \\ 究}

任常兴 ${ }^{1}$, 李海涛 ${ }^{2}$, 周宁 ${ }^{2}$, 袁雄军 ${ }^{2}$

1 公安部天津消防研究所 天津 300381

2 常州大学 石油工程学院 江苏省油气储运技术省重点实验室 江苏 常州 213016

\begin{abstract}
According to the urgent require and hot issues on the region accidents risk and management in chemical industry park, some accident cases at home and abroad were collected and classfied by the accident types. Based on the accident theory of chain of accident caused, the evolution basic parameters and analysis method of event tree, the evolution process of all types of accidents about chemical industry park were described and analyzed, and the risk management system with GIS was put forward. This study is helpful to improve the scientific decision-making and handling ability of safety production accident in the chemical industry park.
\end{abstract}

Key words: Chemical Industry Park; accident risk analysis; risk management; GIS system

摘要

针对当前化工园区内区域安全生产事故 风险的突出问题和风险管治的迫切需求, 通过 搜集国内外化工园区事故案例, 分析归纳出化 工园区主要事故类型; 结合事故连锁致因理论、 事故演化基本参数及事件树分析方法, 对化工 园区各类事故演化过程进行了系统研究, 并初 步构建了基于 GIS 系统的风险管控体系。该研 究有助于提升基于区域事故风险分析的化工 园区安全生产事故应急处置及辅助决策能力。 关键词: 化工园区; 事故风险分析; 风险管理; GIS 系统

\section{引言}

统计表明, 近十多年来我国化工行业火灾、 爆炸事故总体呈上升趋势, 一些大型石油化工
企业的火灾、爆炸事故造成极大的人员伤亡及 财产损失。伴随着化学工业向着深加工、集约 化方向的不断发展, 化工园区数量及规模的不 断扩大, 部分企业工艺设备老化, 化工原料储 量倍增, 呈现潜在重大危险源密集、储量大且 品种多样等特点。易燃易爆的化学品生产过程 往往具有高温高压、有毒有害、有腐蚀性等特 点, 一旦发生火灾、爆炸事故就可能形成连锁 灾害事故, 这不仅对园区内设施设备及居民生 命等造成威胁, 而且由火灾、爆炸事故引发的 次生灾害, 将会威胁周边区域的人民生命财产 安全 ${ }^{[1]}$ 。

本文系统分析了化工园区主要事故类型 与事故演化模式, 提出了基于 GIS 风险分析的 事故应急辅助决策系统架构, 有助于提升化学 工业园区风险管控水平和应急救援处置能力, 优化园区总体安全资源规划与配置。

\section{1. 化工园区主要事故类型与模式}

\section{1 化工园区事故类型分析}

化工园区作为化工企业的集聚区, 物料储 运大多是有毒有害或易燃易爆的危化品, 其输 送方式多为管道, 储存形式多为各类型储罐, 工艺过程主要为收发、生产、储运等, 其最主 要的初发事故为容器设备失效泄漏事件 (LOC) ${ }^{[2]}$ 。主要包括: (1)普通 LOC: 普通失效事件涵 盖了所有常见的设备设施失效原因。比如腐蚀、 结构错误、焊接损坏、储罐排气孔的堵塞等; (2)外部冲击 LOC: 由于外部冲击所引起的储 运装置失效事件; (3)装卸 LOC: 主要为将物 料从运输设备运往固定设备或相反过程中的 失效事件; (4)特定的 LOC: 指由于储运设备、 工艺、物料等特定因素造成的失控失效事件, 
比如化学反应或多米诺效应等。

\section{2 化工园区事故模式演化}

根据对园区事故类型的初步分析, 结合事 故连锁致因理论, 对园区重大事故模式进行逻 辑抽象, 将园区事故归纳为因果事件, 即可建 立基于事故演化视角的重大事故模型 ${ }^{[3]}$, 将化 工园区事故分解为事故能量 $(E)$ 、事故成因 $(R)$ 、 事故机理 $(P) 、$ 触发条件 $(\mathrm{T})$ 、失效状态 $(\mathrm{S})$ 、 点火类型 (I)、事故后果 (C) 等事故属性 (因
素), 其模式表征形式为事故后果, 具体模型 如图 1 所示。

通过化工园区事故模式可看出, 如果以事 故后果来表述事故模式, 那么事故后果是事故 能量 $(E)$ 、事故成因 $(R)$ 、事故机理 $(P)$ 、 触发条件 $(T)$ 、失效状态 $(S)$ 、点火类型 $(I)$ 等事故属性的无量纲函数 ${ }^{[4]}$, 见式 (1.1):

$$
C(x)=f\left(E_{i} ; R_{\mathrm{j}} ; P_{k} ; S_{l} ; T_{m} ; I_{n}\right)
$$

式中: $C(x)$ 一事故后果模式, $x \in[1,14]$ 。

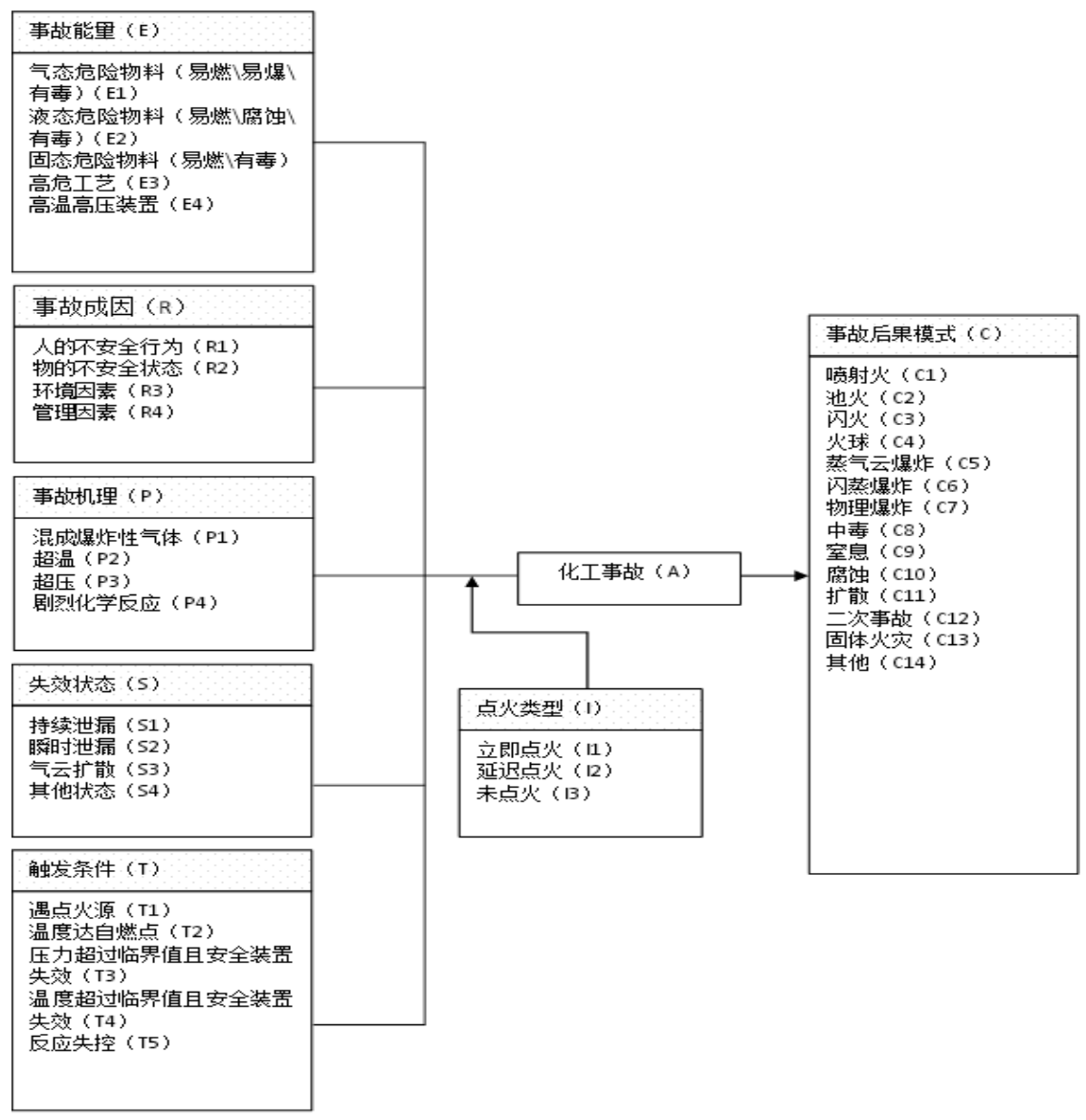

图 1 化工园区事故模式演化

\section{2. 化工园区事故基本参数表征}

\section{1 化工园区事故演化基本参数}

化工园区事故演化机理的基本参数主要 包括物质特性分类、初始事件、初始事件场景、
中间演化事件和事故后果等, 基本参数见表 1 所示。

\section{2 基于事件树的化工园区事故演化过程}

化工园区的固有风险在于储存有大量的 
Risk Analysis and Crisis Response in Big Data Era (RAC-16)

表 1 危险化学品事故演化机理基本参数

\begin{tabular}{|c|c|c|c|c|c|}
\hline \multicolumn{2}{|c|}{ 物质特性分类 } & 初始事件 & 初始事件场景 & 中间演化事件 & 事故后果 \\
\hline \multirow{5}{*}{ 液体 } & 低挥发性、易燃 & 液体泄漏 & -- & 点火 & 池火灾、无后果 \\
\hline & $\begin{array}{l}\text { 低挥发性、易燃、 } \\
\text { 有毒 }\end{array}$ & 液体泄漏 & -- & 点火 & 池火灾、毒物扩散 \\
\hline & 高挥发性、易燃 & 液体泄漏 & -- & $\begin{array}{l}\text { 立即点火、延迟 } \\
\text { 点火、火焰加速 }\end{array}$ & $\begin{array}{l}\text { 池火灾、爆炸、闪火、 } \\
\text { 无后果 }\end{array}$ \\
\hline & $\begin{array}{l}\text { 高挥发性、易燃、 } \\
\text { 有毒 }\end{array}$ & 液体泄漏 & - - & 点火 & 池火灾、毒物扩散 \\
\hline & 有毒液体 & 液体泄漏 & - & - - & 毒物扩散、无后果 \\
\hline \multirow{8}{*}{ 气体 } & \multirow[b]{2}{*}{$\begin{array}{l}\text { 高度易燃、加压 } \\
\text { 液化 }\end{array}$} & 两相泄漏 & 持续泄漏 & $\begin{array}{l}\text { 立即点火、延迟 } \\
\text { 点火、火焰加速 }\end{array}$ & $\begin{array}{l}\text { 池火灾、喷射火、爆炸、 } \\
\text { 闪火、无后果 }\end{array}$ \\
\hline & & $\begin{array}{l}\text { 液体或气 } \\
\text { 溶胶泄漏 }\end{array}$ & 瞬时泄漏 & $\begin{array}{l}\text { 立即点火、火 } \\
\text { 球、延迟点火、 } \\
\text { 火焰加速 }\end{array}$ & $\begin{array}{l}\text { 火球、爆炸、气云火灾、 } \\
\text { 闪火、无后果 }\end{array}$ \\
\hline & 低温冷冻、易燃 & 液体泄漏 & - & $\begin{array}{l}\text { 立即点火、延迟 } \\
\text { 点火、火焰加速 }\end{array}$ & $\begin{array}{l}\text { 池火灾、爆炸、闪火、 } \\
\text { 无后果 }\end{array}$ \\
\hline & 易燃、压缩 & 气体泄漏 & 持续泄漏 & 点火 & 喷射火、无后果 \\
\hline & 易燃、有毒 & 气体泄漏 & 持续泄漏 & 点火 & 喷射火、毒物扩散 \\
\hline & 有毒 & 气体泄漏 & 持续、瞬时泄漏 & -— & 毒物扩散 \\
\hline & \multirow[b]{2}{*}{$\begin{array}{l}\text { 高度易燃、加压 } \\
\text { 液化、有毒 }\end{array}$} & 液体泄漏 & 持续泄漏 & 立即点火 & $\begin{array}{l}\text { 池火灾、喷射火、毒物 } \\
\text { 扩散 }\end{array}$ \\
\hline & & $\begin{array}{l}\text { 液体（气 } \\
\text { 溶胶）泄 } \\
\text { 漏 }\end{array}$ & 瞬时泄漏 & $\begin{array}{l}\text { 立即点火、火 } \\
\text { 球、延迟点火、 } \\
\text { 火焰加速 }\end{array}$ & $\begin{array}{l}\text { 火球、爆炸、气云火灾、 } \\
\text { 闪火、毒物扩散 }\end{array}$ \\
\hline
\end{tabular}

各类危险化学品物料, 不同的物料有不同的事 故演化模式。依据塞韦索 II 指令关于危化品 分类标准对园区内的危险物料进行分类研究, 并结合事件树分析原理对不同危险物料事故 演过程进行了详细分析 ${ }^{[5-7]}$ 。

（1）低挥发性易燃液体

对于像煤油和苯乙烯之类的易燃、低挥发 性液体物料 (室温时闪点 $\left(T_{\mathrm{f}}\right)$ 范围为 $21^{\circ} \mathrm{C}<$ $\mathrm{T}_{\mathrm{f}} \leqslant 55^{\circ} \mathrm{C}$ ), 事故演化过程主要为点燃 (池火 灾事故) 和未点燃 (无后果事件)。点火概率 参考取值为 $P_{1}=0.01$, 其相应的事件树如图 2.1 所示。因挥发性低而不会形成蒸气云, 可忽略 延迟点火事件, 对于闪点大于 $55^{\circ} \mathrm{C}$ 的低挥发 性易燃液体 (如柴油等), 因被点燃的概率非 常低, 定量风险分析时一般予以忽略。

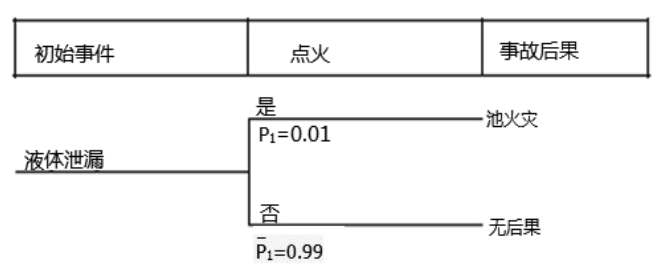

图 2.1 低挥发性易燃液体泄漏事故演化事件树

（2）低挥发性易燃、有毒液体

对于像环氧氯丙烷之类低挥发性易燃、有 毒液体物质事故演化过程类似于 2.1 , 但主要 事故后果增加了毒物质扩散。池火灾发生的概 率仍然为 0.01 , 而毒物扩散的概率为 0.99 , 相比毒物扩散, 池火灾风险在定量风险分析时 可忽略。其通用事件树如图 2.2 所示。 
Risk Analysis and Crisis Response in Big Data Era (RAC-16)

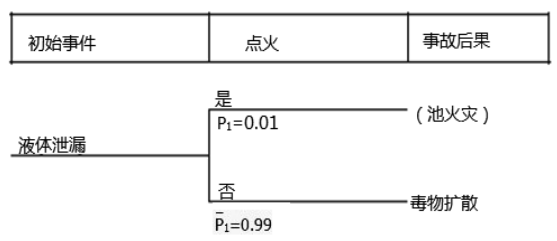

图 2.2 低挥发性易燃、有毒液体泄漏事故演化 事件树

（3）高挥发性、易燃液体

对于像汽油之类的闪点小于 $21^{\circ} \mathrm{C}$ 的高挥 发性、易燃液体而言, 除了立即点火外, 还有 延迟点火导致闪火或爆炸事故。通常, 点燃后 的火焰会发生回火而导致池火灾, 有时会因大 量蒸气云的积聚, 发生火焰加速而导致蒸气云 爆炸事故, 演化过程通用事件树如图 2.3 所示。

立即点火概率取值为 0.065 , 延迟点火概 率取值如下: 处于爆炸极限内的气云扩散至厂 区外为 1 、装置区为 0.7 、防爆区且无点火源 为 0.1 、储存区为 0.01 。爆炸条件为大量易燃 液体泄漏后在空气中挥发形成蒸气云并在爆 炸极限范围内, 如果满足条件则发生爆炸的概 率为 0.4 , 发生闪火概率为 0.6 。如果不能满 足爆炸条件则主要事故后果为闪火, 发生闪火 概率为 1 。而伴随爆炸和闪火事故出现的池火, 因事故后果相比爆炸和闪火较小, 在定量风险 分析时往往忽略。

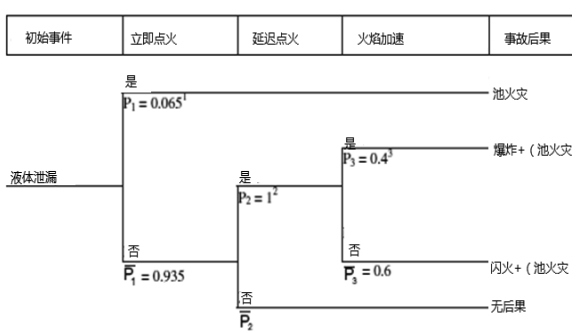

图 2.3 高挥发性、易燃液体泄漏事故演化事 件树

（4）高挥发性、易燃、有毒液体

对于像苯或丙烯腈之类的高挥发性、易燃、 有毒液体物质, 基于易燃性和易挥发性的事故 演化过程类似于 2.3 , 最大的不同在于之前的 无后果在这里为毒物扩散。如果延迟点火的概 率为 $100 \%$, 则毒物扩散事故后果可忽略; 如 果考虑毒物扩散, BEVI 建议将有毒易燃蒸气 认定为 2 种事故后果: 纯粹的火灾 (池火灾)
和纯粹的毒物扩散 (不发生点火事件), 因此 可将通用事件树可简化为图 2.4 (不包括蒸气 云爆炸和闪火事故后果) 所示。

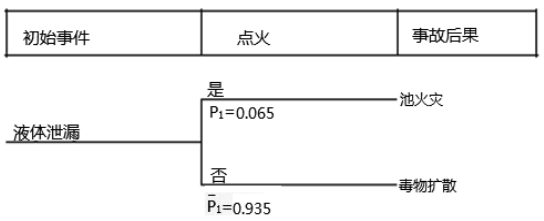

图 2.4 高挥发性、易燃、有毒液体泄漏事故 演化事件树

（5）高度易燃、加压液化气体

\section{1）持续泄漏}

对于像液化丙烷和丁烷之类的极度易燃 气体加压液化后如发生持续泄漏事件, 立即点 火则发生池火灾或喷射火事故 (根据压力的大 小), 延迟点火如遇火焰加速则发生爆炸事故, 否则演化为闪火事故。通用事件树见图 2.5 所 示。

立即点火概率取决于泄漏速率 (泄漏到空 气中的净流量 $): 0.2(<1 \mathrm{~kg} / \mathrm{s}) ; 0.5(1 \mathrm{~kg} / \mathrm{s}$ $\sim 50 \mathrm{~kg} / \mathrm{s}) ; 0.7(>50 \mathrm{~kg} / \mathrm{s})$, 延迟点火概率 一般取值为 1 。延迟点火后如发生火焰加速, 则爆炸概率为 0.4 , 闪火为 0.6 。如果满足泄 漏量达到一定程度（通常指 $500 \mathrm{~kg}-1000 \mathrm{~kg}$ ), 且蒸气云处在爆炸极限范围内, 则发生蒸气云 爆炸事故, 否则事故后果为闪火, 如不满足爆 炸条件则发生闪火的概率为 1 。

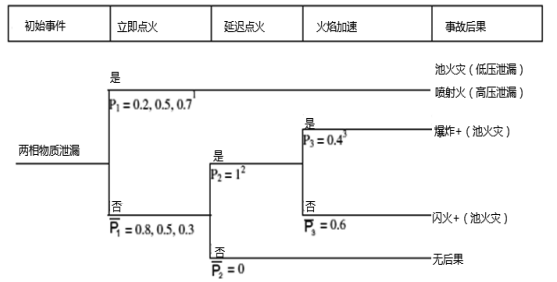

图 2.5 高度易燃、加压液化气体持续泄漏事 故演化事件树

\section{2) 瞬时泄漏}

极度易燃气体加压液化后如发生瞬时泄 漏事件, 立即点火则可演化为火球、爆炸或气 云燃烧等事故后果, 延迟点火如遇火焰加速则 发生爆炸事故, 否则演化为闪火事故。通用事 件树见图 2.6 所示。

立即点火概率取值如下： $0.2(\mathrm{~m} \leqslant 1000$ $\mathrm{kg}) ; 0.5(1000 \mathrm{~kg}<\mathrm{m}<10,000 \mathrm{~kg}) ; 0.7$ 
$(m \geqslant 10,000 \mathrm{~kg})$ 其中 $m$ 为泄漏量。之后发 生火球事故概率取值为 0.7 ; 延迟点火概率一 般取值为 1 , 延迟点火后如发生火焰加速, 则 爆炸概率为 0.4 , 闪火为 0.6 。

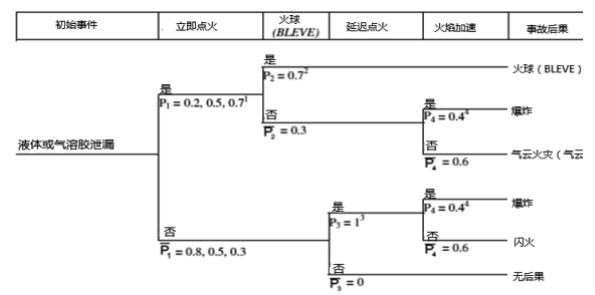

图 2.6 高度易燃、加压液化气体瞬时泄漏事 故演化事件树

（6）高度易燃加压液化、有毒气体

1) 持续泄漏

对于像环氧乙烷之类的高度易然有毒气体加 压液化后如发生持续泄漏初始事件, 应当考虑 毒物扩散事故后果, 忽略延迟点火事件场景, 因为相对而言毒物扩散后果更为严重, 此时闪 火事故后果可不考虑。将此事故演化过程简化 为火灾 (池火和喷射火) 和毒物扩散 2 种事故 后果, 演化通用事件树见图 2.7 所示。

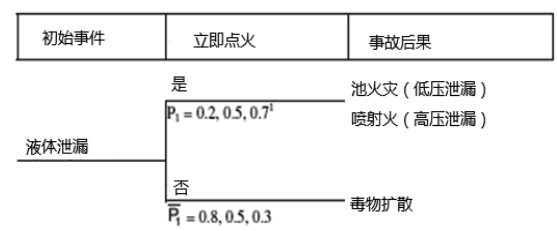

图 2.7 高度易燃加压液化、有毒气体持续泄漏 事故演化事件树

2) 瞬时泄漏

高度易燃加压液化、有毒气体如发生瞬时 泄漏初始事件时, 立即点火可导致火球 (BLEVE)、爆炸和气云火灾等事故后果, 未点 火则演化为毒物扩散事故, 概率取值具体见图 2.8 所示。

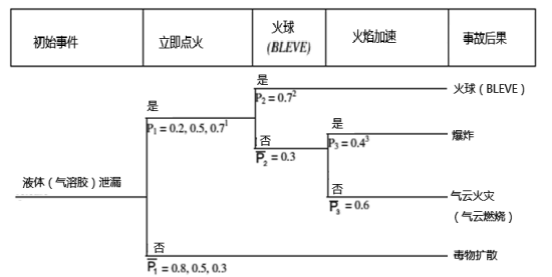

图 2.8 高度易燃加压液化、有毒气体瞬时泄 漏事故演化事件树

\section{3. 化工园区应急管理体系}

\section{1 定量化风险管理}

目前安全管理模式逐步由 “事故应对型” 向 “风险管理型” 转变, 强调以防为主, 积极 控制的安全管理模式, 重点分析事故肇因、不 断吸取事故经验教训 ${ }^{[8]}$ 。

定量化风险管理是通过辨识系统中的各 类潜在危险源, 计算分析后确定事故发生概率 的大小以及事故后果的严重程度, 根据事故监 测的参数和预设的报警阈值, 采取有效的监测 预警措施和风险控制对策, 从而预防事故发生 或降低事故后果的严重程度。

\section{2 基于 GIS 的风险管理系统}

基于GIS的风险管理系统使定量化风险管 理技术得到了质的提升。目前GIS系统开发研 究的种类众多, 在化工园区风险管理中可实现 对园区危险源信息的查询、园区危险源与生产 工艺的实时监控与事故监测预警、园区应急救 援设备设施的集约式管理、园区内重大事故模 拟与后果监测、根据事故现场信息提供最佳的 救援与疏散路径、火灾事故应急联动以及强大 的数据库教育培训功能等 ${ }^{[9-11]}$ 。

通过对化工园区内重大危险源的事故风 险分析, 结合强大的GIS系统, 采用视频监控、 温度、浓度、压力等传感器对园区危险源进行 实时监控。系统对采集到的监控数据进行显示、 记录和分析, 当工艺参数超过规定值时系统自 动声、光报警 (监控室报警和现场报警), 并 显示监控参数超标危险源或工艺的具体信息 （如企业名称, 超标危险源或工艺的名称、位 置, 建议处置措施等) ${ }^{[12]}$ 。一旦遭遇危化品火 灾、泄漏、爆炸等事故, 系统首先对各事故进 行自动模拟分析, 确定事故的影响范围和后果 并进行危险度等级评定, 根据事故危险度等级 企业单位可做出相应的应急处置方案。如果遇 到重大火灾爆炸事故, 系统可实现对各种消防 救援资源设备信息的数字化, 利于消防救援的 及时性和准确性, 并根据事故现场信息提供最 佳的救援与疏散路径, 便于相关人员开展应急 救援工作。

\section{3 风险预测预警与应急辅助决策体系}

结合GIS系统, 建立化工园区火灾风险预 测预警与应急辅助决策体系, 完整体系主要包 括基础信息模块, 安全检查模块、风险分析模 
Risk Analysis and Crisis Response in Big Data Era (RAC-16)

块和事故应急模块四部分。

1) 基础信息模块 该模块根据存储内容的 不同分为基础数据、企业信息以及地理信息三 个部分, 可完成企业基础数据信息维护、地理 信息维护、危险作业维护以及有害因素维护等。 为了减少数据录入的工作量, 企业基础信息还 可以通过外部Exce1或者Access文件直接导入 此外企业信息还可以按照用户的需求导出至 Exce1文件中。

2) 事故监测预警模块 该模块主要是采用 视频监控设备以及各种功能的传感器设备对 高危险工艺、重大危险源以及移动危险源等进 行实时监测, 并将初步监测结果发送至监测预 警主机, 当监测值超过预警阈值时监控预警主 机自行给出报警信号、并向短信发送设备发出 事故应急命令、将监控信息发送至事故模拟模 块, 预测事故后果。
3) 风险分析模块 风险分析模块包括危险 工艺分析、危险作业分析、特种设备以及重大 危险源分析, 可完成工艺危险特点及危险程度 判断、应急处置方案及控制方式实现、事故伤 害范围以及风险等级评估等。

4) 事故应急辅助决策模块 事故应急辅助 决策模块包括应急救援体系、应急资源信息、 事故应急处置、救援法律法规、危化品信息、 应急装备与资源查询。系统根据监测信息确定 事故发生的位置, 系统预测事故发展趋势及后 果, 系统自动生成应急辅助决策方案, 方案包 括事故企业信息、事故类型、涉及的危化品信 息、最佳救援疏散路径、附近的应急资源、应 急力量分布、建议的事故扑救措施、专家信息 等等, 该应急辅助决策方案可为应急指挥人员 提供科学的决策依据, 提高救援效率。架构建 立如图 3.1所示。

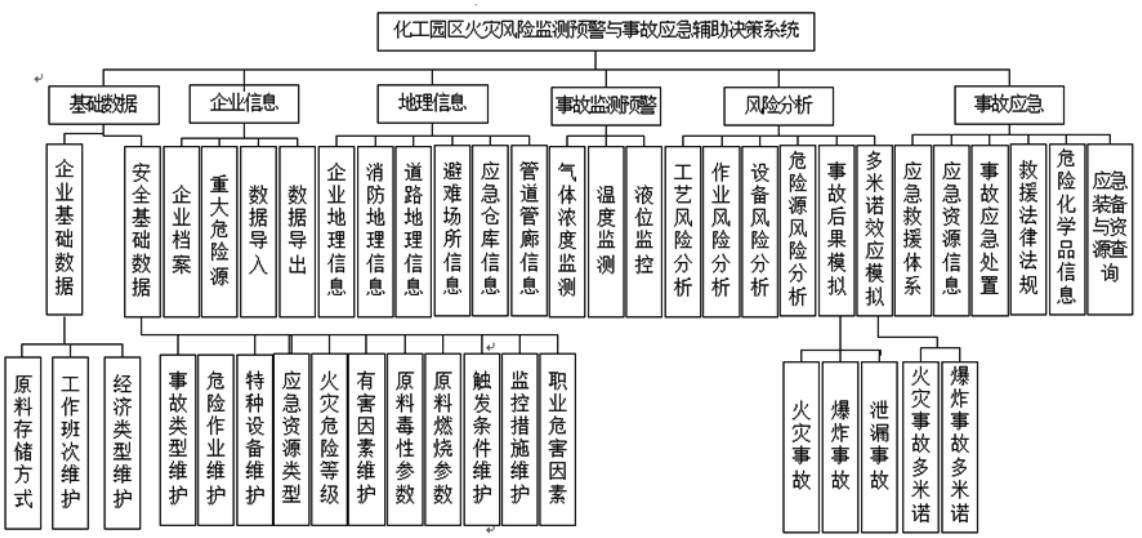

图3.1 化工园区应急管理体系功能结构图

\section{4. 结束语}

本文通过搜集国内外化工园区各类事故 案例, 总结分析出化工园区主要的初发事故为 容器设备失效泄漏事件 (LOC), 并藉此对事故 类型进行具体划分; 根据事故演化机理及基本 参数和事件树分析方法对各类事故的演化趋 势进行了详细剖析, 并初步构建了基于GIS系 统的风险管理体系, 建立了化工园区应急管理 体系架构。该研究对提升区域风险管治和科学 化应急处置具有一定的指导意义。

\section{参考文献}

[1] L.A. Jordan, BP's Reputation Repair
Strategies during the Gulf Oil Spill . Journal of Risk Analysis and Crisis Response, 2015, $5(1): 2-15$.

[2] 任常兴, 李晋, 孙晓涛等. 火灾高危单 位消防安全评估探. 风险分析和危机反应 中的信息技术, 黄崇福, 包玉海, 赵思健 编。巴黎：Atlantis 出版社, 2014. 725-729.

[3]刘暄亚. 大型石油储罐区火灾风险预测预 警技术研究. 消防科学与技术, 2012, 31(2):193-195.

[4]张永强, 相艳景, 毛星等. 多米诺效应的 风险分析方法。安全与环境工程, 
Risk Analysis and Crisis Response in Big Data Era (RAC-16)

2008,8(6):152-159.

[5] 刘茂. 事故风险分析理论与方法 $[M]$. 北 京: 北京大学出版社, 2011

[6] 袁雄军, 任常兴. 危险化学品泄漏事故点 火概率取值研究. 中国安全科学学 报, 2011,21(9): 39-46.

[7] Ronza, A., Vílchez, J. A., Casal, J..Using transportation accident databases to investigate ignition and explosion probabilities of flammable spills . Journal of Hazardous Materials, 2007, 146(7):106-123.

[8］赵文芳, 武志峰. 重大危险源定量风险评 估技术研究. 安全健康与环 境, 2012,2(12):1-5.
[9]李俊华, 刘俊, 周宁. 基GIS热辐射事故多米 诺叠加分析与设计, 东华大学学报, 2012.38(6):728-731

[10］徐士会，周宁. 基于GIS的化工园区风险 管理信息系统研究. 常州大学学报, 2012,24(4):73-75.

[11] M.T. Ding, C. Miao, GIS-based Assessment of Vulnerability to Landslide Hazards in Lushan Earthquake-stricken Areas. Journal of Risk Analysis and Crisis Response, 2015, 5(2), 93-106.

[12］李俊华. 基于GIS的化工园区风险及应 急管理系统分析与设计. 江苏大学, 2013. 